\title{
Overview of the 2018 Workshop on Iterative Errors in Unsteady Flow Simulations
}

\author{
L. Eça* \\ MARIN Academy and IST ULisboa \\ Wageningen Netherlands and Lisboa Portugal \\ Email: luis.eca@ist.utl.pt \\ G. Vaz \\ WaVEC Offshore Renewables \\ Lisboa Portugal \\ Email: guilherme.vaz@wavec.org \\ M. Hoekstra \\ S. Pal \\ E. Muller, D. Pelletier \\ Consultant \\ ASME Member \\ Ecole Polytechnique Montreal \\ Voorthhuizein The Netherlands Herndon, U.S.A. \\ Montreal Canada
}

\begin{abstract}
A. Bertinetti, R. Difonzo, L. Savoldi, R. Zanino and A. Zappatore Y. Chen, K.J. Maki, H. Ye
Dipartimento Energia "Galileo Ferraris" Politecnico di Torino

Torino Italy

University of Michigan

Michigan U.S.A.
\end{abstract}

\author{
Jernej Drofelnik, Benjamin Moss, Andrea Da Ronch \\ University of Southampton \\ Southampton United Kingdom
}

\begin{abstract}
Two Workshops were held at the ASME V\&V Symposiums of 2017 and 2018 dedicated to Iterative Errors in Unsteady Flow Simulations. The focus was on the effect of iterative errors on numerical simulations performed with implicit time integration, which require the solution of a non-linear set of equations at each time step. The main goal of these Workshops was to create awareness to the problem and to confirm that different flow solvers exhibited the same trends.

The test case was a simple two-dimensional, laminar flow of a single-phase, incompressible, Newtonian fluid around a circular cylinder at the Reynolds number of 100. A set of geometrically similar multi-block structured grids was available and boundary conditions to perform the simulations were proposed to the participants. Results from seven flow solvers were submitted, but not all of them followed exactly the proposed conditions. One set of results was obtained with adaptive grid and time refinement using triangular elements (CADYF) and another used a compressible flow solver with a dual time stepping technique and a Mach number of 0.2 (DLR-Tau). The remaining five submissions were obtained with five different incompressible flow solvers
\end{abstract}

*Address all correspondence related to ASME style format and figures to this author. 
(ANSYS CFX 14.5, pimpleFoam, ReFRESCO, SATURNE, STAR CCM+v12.06.010-R8) using implicit time integration in the proposed grids.

The results obtained in this simple test case showed that iterative errors may have a significant impact on the numerical accuracy of unsteady flow simulations performed with implicit time integration. Iterative errors can be significantly larger (one to two orders of magnitude) than the residuals and/or solution changes used as convergence criteria at each time step. The Courant number affected the magnitude of the iterative errors obtained in the proposed exercise. For the same iterative convergence criteria at each time step, increasing the Courant number tends to increase the iterative error.

\section{Introduction}

Simulation of (statistically) unsteady flows is no longer an exceptional task in Engineering applications of Computational Fluid Dynamics (CFD). For wall-bounded (turbulent) flows at high Reynolds numbers it is usual to apply implicit techniques for time integration 1 , i.e. a non-linear system of partial differential equations must be solved at each time step. It should be pointed out that implicit time integration is not restricted to the use of the Reynolds-Averaged Navier Stokes (RANS) equations. It is also usually applied in Large-Eddy Simulations (LES) and with hybrid RANS/LES or bridging models, as for example Detached Eddy-Simulation (hybrid) or Partially-Averaged Navier Stokes equations (PANS, bridging). Therefore, when implicit time integration is adopted, a convergence tolerance is needed to decide on when having solved this non-linear system well enough. Any iterative error propagates to the next time step and so the numerical error of a given solution may not be dominated by the numerical error inherent to the space and time discretization. In fact, it has been recently shown using a onedimensional manufactured solution [1] that in an unsteady flow simulation dominated by iterative errors it is pointless to refine the grid and/or the time step.

Two Workshops dedicated to Iterative Errors in Unsteady Flow Simulations were held at the ASME V\&V Symposiums of 2017 and 2018 [2,3]. The main goal of these Workshops was to create awareness to the problem and to confirm that different flow solvers exhibited the same trends. The simple twodimensional, laminar flow of a single-phase, incompressible, Newtonian fluid around a circular cylinder is sufficient to illustrate the challenges posed by iterative errors in unsteady flow simulations. There is no attempt to perform Validation (comparison with experimental results) or derive a reference solution for the selected test case to estimate discretization errors (Solution Verification).

The determination of iterative errors does not require the knowledge of the experimental solution or the exact solution of the Navier-Stokes equations. The best estimate of the iterative error, requires a solution obtained for a given grid and time step with the non-linear residual at each time step reduced to machine accuracy (using double precision) and a sufficient simulation time to reduce statistical errors (effects of the initial condition) also to machine accuracy. This reference solution depends on the space and time discretization techniques used by each flow solver and so it is not possible to generate a solution valid for any CFD solver using the same grid and time step. On the other hand, even for the simple flow proposed for this exercise, generating this reference solution is too expensive or sometimes even impossible. Therefore, iterative errors are addressed by comparing the solution of selected integral and local flow quantities (described below) obtained with the same simulation settings and with at least three different levels of the iterative convergence criterion applied at each time step.

For both Workshops, a set of four geometrically similar multi-block structured grids was available and boundary conditions to perform the simulations were proposed to the participants. However, in the 2017 edition two Reynolds numbers based on the velocity of the uniform incoming flow $V_{\infty}$, cylinder diameter $D$ and kinematic viscosity $v$ of $R e=100$ and $R e=150$ were proposed to the participants.

\footnotetext{
${ }^{1}$ If the fluid is incompressible a system of equations must be solved even for explicit time integration.
} 
Furthermore, the time step was not imposed and so there were almost no simulations performed with the same settings by different flow solvers. In the 2018 edition, only $R e=100$ was addressed and four different dimensionless time steps were proposed for each grid, corresponding to four grid/time refinements at fixed Courant number. As a consequence, the submitted results obtained from seven different flow solvers (ANSYS CFX 14.5 [4], CADYF [5], DLR-Tau [6], pimpleFoam [7], ReFRESCO [8], SATURNE [9] and STAR CCM+ v12.06.010-R8 [10]) included plenty of cases with the same flow conditions. Therefore, the present paper focuses only on the 2018 edition of the Workshop.

The remainder of this paper is organized in the following way: section 2 presents the proposed test case including the computational domain, boundary conditions and selected quantities of interest; the proposed grids and time steps are described in section 3, whereas section 4 contains a brief description of the seven flow solvers used in this exercise together with the iterative convergence criteria used at each time step; section 5 illustrates and discusses the results and the main conclusions of this Workshop are summarized in section 6.

\section{Test Case}

\subsection{Domain and boundary conditions}

The selected test case is the two-dimensional, laminar flow around a circular cylinder at Reynolds number $\left(R e=\frac{V_{\infty} D}{v}\right)$ of $R e=100$. It was suggested to assume that the fluid is incompressible (Mach number equal to 0 ), but there was a submission using Mach number of 0.2 at the inlet.

The calculation domain is a rectangle with the distances of the boundaries to the cylinder centre equal to $20 \mathrm{D}$ upstream for the inlet, $80 \mathrm{D}$ downstream for the outlet and $40 \mathrm{D}$ for top and bottom boundaries, as illustrated in figure 1 . The Cartesian $(x, y)$ coordinate system adopted has the origin at the centre of the cylinder and the $x$ axis aligned with $V_{\infty}$. Since the focus of the exercise is on numerical errors (especially on iterative errors) and not on comparisons with experiments, there is no need to make any sensitivity study to justify these choices. Nonetheless, the boundaries must be sufficiently away from the cylinder to ease the selection of boundary conditions.

The simulation of an unsteady flow requires the specification of an initial condition. In the present test case, the flow becomes periodic with a period/frequency related to the vortex shedding in the wake of the cylinder. The present exercise focus on the periodic part of the flow and so the initial condition will only affect the simulation time required to attain a periodic flow.

The proposed boundary conditions are the following: no-slip and impermeability conditions at the cylinder surface $\left(\sqrt{x^{2}+y^{2}}=D / 2\right)$ and normal derivative of the pressure set equal to zero $(\nabla p \cdot \vec{n}=0)$; uniform flow at the inlet boundary $(x=-20 D)$ with horizontal velocity component $V_{x}=V_{\infty}$ and vertical velocity component $V_{y}=0$; pressure imposed at the outlet boundary $\left.\right|^{2}(x=80 D)$ and derivatives of the two velocity components in the streamwise direction $(x)$ equal to zero $\left(\frac{\partial V_{x}}{\partial x}=\frac{\partial V_{y}}{\partial x}=0\right)$; free-slip at the top and bottom boundaries $(y= \pm 40 D)$, i.e. normal velocity component equal to zero $\left(V_{y}=0\right)$ and derivatives of the velocity component in the horizontal direction and pressure in the normal direction equal to zero $\left(\frac{\partial V_{x}}{\partial y}=\frac{\partial p}{\partial y}=0\right)$.

\subsection{Quantities of interest}

Integral/functional and local flow quantities on the cylinder surface were selected for the assessment of the iterative errors. The selected quantities of interest are related to the time history of the lift $C_{L}$ and

\footnotetext{
${ }^{2}$ Pressure streamwise derivative equal to zero may also be used as a boundary condition, but in that case pressure level must be fixed at a point.
} 
drag $C_{D}$ coefficients or to the time-averaged pressure $C_{p}$ and skin friction $C_{f}$ coefficients on the cylinder surface. The lift and drag coefficients are obtained from

$$
C_{L}(t)=\frac{F_{L}(t)}{\frac{1}{2} \rho V_{\infty}^{2}}, C_{D}(t)=\frac{F_{D}(t)}{\frac{1}{2} \rho V_{\infty}^{2}} .
$$

where the vertical $L$ and horizontal $D$ forces are calculated from the integration of the pressure $p$ and shear-stress $\tau_{w}$ on the cylinder surface and $\rho$ is the density of the fluid. The pressure and skin friction coefficients are obtained from

$$
C_{p}(\theta, t)=\frac{p(\theta, t)-p_{\infty}}{\frac{1}{2} \rho V_{\infty}^{2}}, C_{f}(\theta, t)=\frac{\tau_{w}(\theta, t)}{\frac{1}{2} \rho V_{\infty}^{2}}
$$

where $p_{\infty}$ is the maximum pressure at the inlet boundary and $\theta=\operatorname{atan}(y / x)+\pi(\theta=0 \equiv(x=-0.5 D, y=0))$.

The integral/functional quantities of interest are:

- The average ${ }^{3} \operatorname{drag}\left(C_{D}\right)_{\text {avg }}$ coefficient

$$
\left(C_{D}\right)_{a v g}=\frac{\int_{0}^{n T} C_{D}(t) d t}{n T}
$$

where $T$ is the period of the lift time history and $n$ is the number of cycles ( $n \geq 4)$ used to determine the average value. The beginning of the cycle corresponds to the instant where $C_{L}=0$ when the lift coefficient is increasing.

- The maximum drag $\left(C_{D}\right)_{\max }$ and lift $\left(C_{L}\right)_{\max }$ coefficients.

- The standard deviation of the drag $\left(C_{D}\right)_{s t d}$ and lift $\left(C_{L}\right)_{s t d}$ coefficients.

$$
\left(C_{D}\right)_{s t d}=\sqrt{\frac{\int_{0}^{n T}\left(C_{D}(t)-\left(C_{D}\right)_{a v g}\right)^{2} d t}{n T}},\left(C_{L}\right)_{s t d}=\sqrt{\frac{\int_{0}^{n T}\left(C_{L}(t)-\left(C_{L}\right)_{a v g}\right)^{2} d t}{n T}} .
$$

\footnotetext{
${ }^{3}\left(C_{L}\right)_{\text {avg }}$ is supposed to be zero.
}

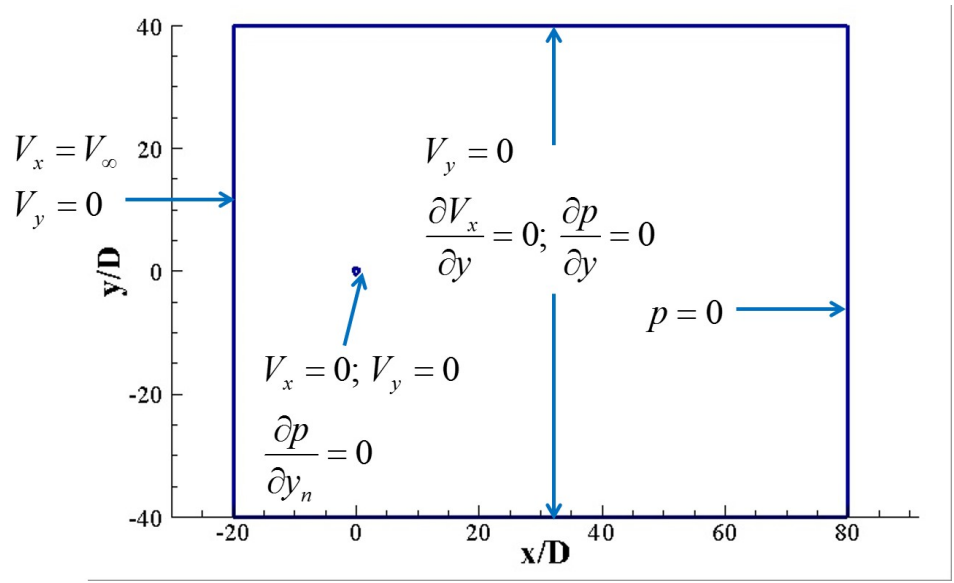

Fig. 1. Illustration of the calculation domain and boundary conditions for the flow around a circular cylinder. 

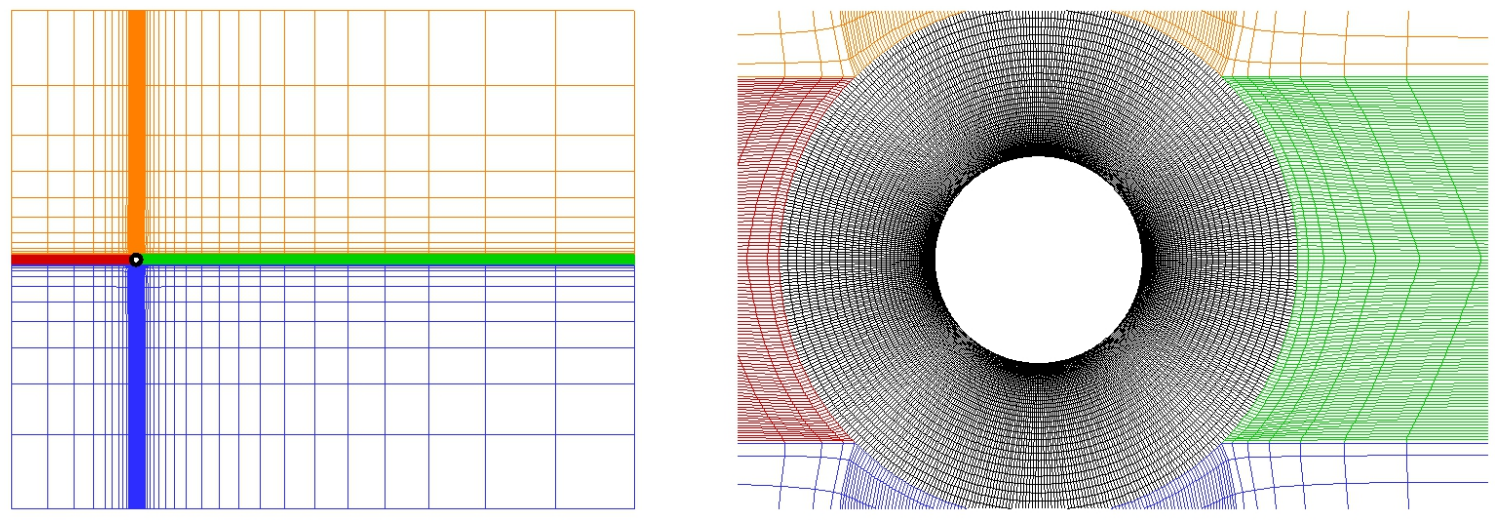

Fig. 2. Illustration of the grids for the calculation domain of the flow around a circular cylinder.

Also derived from $C_{L}(t)$, the Strouhal number $S t$

$$
S t=\frac{D}{T V_{\infty}}=\frac{D f}{V_{\infty}}
$$

where $f$ is the frequency of the vortex shedding, $f=1 / T$.

The local flow quantities are the time-averaged base pressure coefficient $\left(C p_{b}\right)_{a v g}$ and the angle of flow separation $\left(\theta_{\text {sep }}\right)_{\text {avg }}$, obtained from

$$
\left(C p_{b}\right)_{a v g}=\frac{\int_{0}^{n T} C p_{b}\left(\theta_{b}, t\right) d t}{n T},\left(\theta_{\text {sep }}\right)_{a v g}=\frac{\int_{0}^{n T} \theta_{s e p}(t) d t}{n T},
$$

where $\theta_{b}=\pi$ and $\theta_{\text {sep }}$ is the $\theta$-coordinate of the point on the upper surface of the cylinder where the wall shear-stress vanishes, $\tau_{w}=0$.

\section{Grids and time step}

There are four geometrically similar multi-block structured grids available, one of them being illustrated in figure 2. The grids have clustering of grid nodes in the block of the near-wake. As a consequence, there is a sudden change of cell size at the edge of the inner block, but several preliminary tests were done to guarantee that these grids are suitable for the determination of the quantities of interest. Furthermore, these tests showed that it was important to include a significant increase of the cell-size towards the outlet, top and bottom boundaries to avoid pressure reflections. Table 1 presents the total number of cells, the number of faces on the cylinder surface and the grid and time refinement ratios $r_{i}=h_{i} / h_{1}=\Delta t_{i} / \Delta t_{1}$ of the four grids as well as the dimensionless time steps with the corresponding maximum values of the Courant number $\mathrm{Co}_{\max }$.

One of the submissions (CADYF) did not use the proposed grids, because this flow solver relies on grid (and time) adaptive refinement [5]. Simulations were performed using a fixed isotropic grid for all simulated times or with mesh adaptation cycles at user-supplied times. However, as discussed below, the iterative convergence criterion used at each time step will affect the adaptive time step. Therefore, its effect on the numerical error of the simulations will be significantly different from the remaining flow solvers that use a fixed time step. In the presentation of CADYF results we will restrict ourselves to those obtained in the adapted fixed isotropic grid, illustrated in figure 3. The final grid is composed of 184884 elements and 370638 nodes with the ratio between the largest and smallest elements approximately equal to 1000 . 


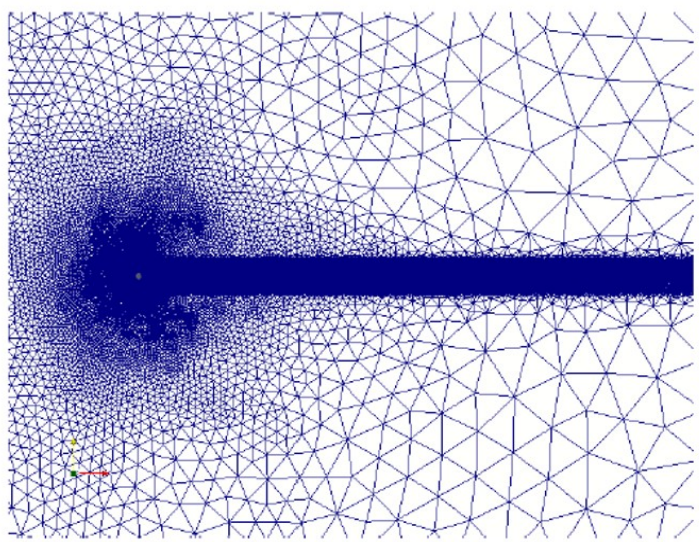

(a) Full view

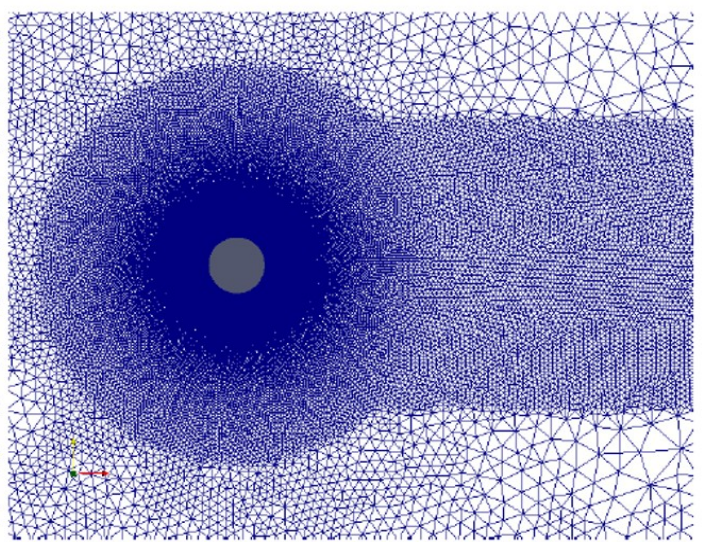

(b) Cylinder vicinity

Fig. 3. Illustration of the adapted fixed isotropic grid used with CADYF for the calculation domain of the flow around a circular cylinder.

\section{Flow solvers and iterative convergence criteria}

Seven flow solvers were used to obtain the results submitted to this Workshop: ANSYS CFX 14.5 [4], CADYF [5], DLR-Tau [6], pimpleFoam [7], ReFRESCO [8], SATURNE [9] and STAR CCM+ v12.06.010-R8 [10]. A brief description of the main features of each solver is given below including the iterative convergence criteria used to perform the simulations.

ANSYS CFX 14.5 [4] is a commercial CFD package based on a finite volume discretization with a fully-coupled solution and Picard linearization. The present simulations using implicit time integration and a fixed time step were performed with second-order space and time discretization schemes. Linear systems of equations were solved with an algebraic multigrid method for linear solvers. Iterative convergence criteria at each time step was controlled by the RMS of normalized residuals, "conservation imbalance" and a maximum of 10 iterations per time step.

CADYF [5] uses a finite element approach with a fully-coupled solution and Newton linearization. The space discretization uses P2-P1 Taylor-Hood elements and the linear systems of equations are solved with the direct solver PARDISO [11]. The implicit time-integrators of order 1 to 5 use an adaptive procedure to update both the time-step size and the order of the integration method at the end of each time iteration. This means that time integration is performed with a variable time step and a variable order of time convergence. Iterative error is controlled by relative changes of solution variables and residuals. It must be pointed out that the iterative error is embedded in the error estimation of the time adaption. Therefore, its effect leads to changes in the time integration scheme due to contributions of the iterative error to the estimated error.

Table 1. Grids available for the Workshops on Iterative Errors in Unsteady Flow Simulations and recommended time steps.

\begin{tabular}{|c|c|c|c|c|c|c|c|}
\hline Grid & \multirow{2}{*}{$\begin{array}{c}\text { Number } \\
\text { of Cells }\end{array}$} & $\begin{array}{c}\text { Number of Faces on } \\
\text { Cylinder Surface }\end{array}$ & $h_{i} / h_{1}$ & \multicolumn{3}{|c|}{ Dimensionless time step, $\Delta t_{i} V_{\infty} / D$} \\
\cline { 4 - 7 } & & & $\mathrm{Co}_{\max }=0.8$ & $\mathrm{Co}_{\max }=1.6$ & $\mathrm{Co}_{\max }=6.2$ & $\mathrm{Co}_{\max }=15$ \\
\hline 4 & 125910 & 960 & 2 & 0.0025 & 0.005 & 0.02 & 0.05 \\
2 & 196266 & 1200 & 1.6 & 0.002 & 0.004 & 0.016 & 0.04 \\
1 & 282174 & 1440 & $1.3(3)$ & $0.0016(6)$ & $0.003(3)$ & $0.013(3)$ & $0.03(3)$ \\
\hline
\end{tabular}


DLR-Tau [6] is a compressible flow solver based on a finite volume discretization that uses a dualtime step approach. With this solver, Mach $=0.2$ has been imposed at the inlet of the computational domain and the fluid is assumed to be a perfect gas. Simulations are performed with second-order time and space discretization techniques. Time integration is performed with an explicit 4-stage RungeKutta scheme using a fixed time step. Iterative errors are related to the convergence of the dual timestep technique and is controlled by the normalized $\rho$ residual using the reference value from the initial approximation at each time step.

pimpleFoam is part of the OpenFoam library [7]. It uses a finite volume discretization with Picard linearization and a segregated solution using a combination of the PISO and SIMPLE algorithms. Simulations were performed with second-order space and time schemes and an implicit time integration with imposed Courant number, which means that the time step changes along the simulation. Linear systems of equations are solved with Bi-CG with ILU preconditioning for momentum, combined with diagonalbased incomplete Cholesky and Gauss-Seidel solver along with a geometric agglomerated algebraic multigrid method for pressure. Iterative errors are controlled by the number of iterations performed at each time step.

ReFRESCO [8] is a community-based CFD solver based on a finite volume discretization and Picard linearization. It can use segregated or coupled approaches and mass conservation is ensured using the SIMPLE algorithm. Simulations were performed with second-order schemes in space and with implicit time integration using second and first-order schemes. Linear systems of equations are solved with GMRES for the momentum equations and CG for the pressure using Jacobi preconditioning in both cases. Iterative error is controlled by the maximum of normalized residuals, which are equivalent to dimensionless dependent variables changes in a simple Jacobi iteration.

SATURNE "is the free, open-source software developed and released by EDF to solve computational fluid dynamics (CFD) applications" [9] that uses a finite volume discretization and Picard linearization. In the present simulations, a coupled solution is used for the velocity components and the SIMPLEC algorithm is applied to ensure mass conservation. Second-order schemes in space are applied and implicit time integration is performed with a first-order scheme and a fixed time step. Linear systems of equations are solved using Jacobi solver for momentum, and AMG method based on CG solver with Jacobi preconditioning for pressure. Iterative errors are controlled by the global sum of the squares of residuals.

STAR CCM+ v12.06.010-R8 is a commercial CFD package [10] based on a finite volume discretization and Picard linearization. Simulations were performed with a segregated approach and a SIMPLE-type algorithm. Space discretization is based on second-order schemes and implicit time integration uses second-order schemes and a fixed time step. An AMG method is used in the solution of the linear systems of equations. As for pimplefoam, iterative errors are controlled by the fixed number of iterations performed at each time step.

\section{Results}

\subsection{General}

As presented in table 1, there were 16 cases proposed to the participants: four grids with four different time steps corresponding to four different values of the maximum Courant number. The number of submissions differed per case, as presented in table 2. Not surprisingly, the largest number of submissions refers to the coarsest grid with the largest time step, the least number to the finest grid with the smallest time step. Table 2 also includes the designation of the time steps proposed for the simulations. For example, case G2TB corresponds to results obtained in grid 2 with time step B $\left(h_{i} / h_{1}=1.3(3)\right)$ with $\Delta t V_{\infty} / D=0.003(3)$ (see table 1 ).

As mentioned above, there was one submission (CADYF) that used grid and time adaptivity which 
Table 2. Submissions for each of the four grids and time steps proposed for the simulation of the flow around a circular cylinder at $R e=$ 100 .

\begin{tabular}{|c|c|c|c|c|}
\hline \multirow{2}{*}{ Grid } & \multicolumn{4}{|c|}{ Dimensionless time step, $\Delta t_{i} V_{\infty} / D$} \\
\cline { 2 - 5 } & $\mathrm{TA}\left(\mathrm{Co}_{\max }=0.8\right)$ & $\mathrm{TB}\left(\mathrm{Co}_{\max }=1.6\right)$ & $\mathrm{TC}\left(\mathrm{Co}_{\max }=6.2\right)$ & $\mathrm{TD}\left(\mathrm{Co}_{\max }=15\right)$ \\
\hline G4 & 4 & 4 & 6 & 7 \\
G3 & 2 & 3 & 6 & 5 \\
G2 & 2 & 3 & 4 & 5 \\
G1 & 1 & 1 & 3 & 4 \\
\hline
\end{tabular}

Table 3. Range of iterative convergence criteria $e_{i t}$ used in the simulation of the flow around a circular cylinder at $R e=100 . N_{s}$ is the number of levels tested. ANSYS-CFX simulations also included a maximum number of iterations at each time step $N_{i t}=10$.

\begin{tabular}{|c|c|c|c|c|}
\hline Solver & $e_{i t}$ & $N_{s}$ & $\varepsilon_{m i n}$ & Test cases \\
\hline ANSYS-CFX & $10^{-5}-10^{-1}$ & 5 & $10^{-4}$ & G4TD \\
CADYF & $10^{-10}-10^{-5}$ & 4 & $10^{-5}$ & "G3TC" \\
DLR-Tau & $10^{-5}-10^{-2}$ & 3 & $10^{-3}$ & All except G1 \\
ReFRESCO S2 & $10^{-8}-5 \times 10^{-3}$ & 9 & $2 \times 10^{-6}$ & All \\
ReFRESCO C2 & $10^{-8}-5 \times 10^{-3}$ & 8 or 9 & $2 \times 10^{-6}$ & All grids for TD and TC \\
ReFRESCO S1 & $10^{-6}-5 \times 10^{-3}$ & 8 & $2 \times 10^{-5}$ & All grids for TD \\
SATURNE & $10^{-10}-10^{-7}$ & 4 & $10^{-3}$ & All grids for TC \\
\hline
\end{tabular}

is therefore not included in the submissions presented in table 2. The results of this submission will be presented with case G3TC. This choice was based on the total number of cells of the final grid and the large number of submissions for G3.

For each of these test cases, each submission includes the results using at least three different levels of the iterative convergence criteria. However, as described above, the seven flow solvers use different techniques to control the iterative error which makes direct comparison difficult. Nevertheless, we have tried to define a strategy that allows to check if all the flow solvers (except CADYF) exhibit the same trends for the influence of the iterative error on the selected quantities of interest. However, the interaction between the convergence of the linear systems equations solved at each non-linear iteration and the non-linear convergence is out of the scope of this study.

The two submissions using the fixed number of iterations at each time step to control the iterative error (pimpleFoam and STAR-CCM+) were asked to determine the average value of the maximum residuals at the end of each time step. This allowed the conversion of number-of-iterations-at-eachtime-step to a representative residual.

For the comparisons of data obtained with different flow solvers, the independent variable $\varepsilon$ that represents the iterative convergence criteria is given by

$$
\varepsilon=\frac{e_{i t}}{\left(e_{i t}\right)_{\max }},
$$


Table 4. Minimum value of the ratio $\varepsilon_{\min }$ of the average residuals obtained with different number of iterations at each time step $N_{i t}$ (pimpleFoam simulations).

\begin{tabular}{|c|c|c|c|c|}
\hline pimpleFoam & G4TD & G3TD & G2TD & G1TD \\
\hline$N_{i t}$ & $4,6,8,20$ & $3,4,6,8,20$ & $2,3,4,6,8$ & $2,3,4,6,8$ \\
$\varepsilon_{\text {min }}$ & 0.037 & 0.094 & 0.0016 & 0.002 \\
\hline
\end{tabular}

Table 5. Minimum value of the ratio $\varepsilon_{\min }$ of the average residuals obtained with different number of iterations at each time step $N_{i t}$ (STAR $\mathrm{CCM}+$ simulations).

\begin{tabular}{|c|c|c|c|c|c|}
\hline STAR CCM+ & Grid & TD & TC & TB & TA \\
\hline$N_{i t}$ & 4 & $10,50,100,200$ & $10,50,100$ & $10,50,100$ & $10,50,100$ \\
$\varepsilon_{\text {min }}$ & & $1.9 \times 10^{-5}$ & $1.2 \times 10^{-4}$ & $1.1 \times 10^{-5}$ & $2.1 \times 10^{-5}$ \\
\hline$N_{i t}$ & 3 & $10,50,100,200$ & $10,50,100$ & $10,50,100$ & - \\
$\varepsilon_{\text {min }}$ & & $3.3 \times 10^{-5}$ & $1.4 \times 10^{-4}$ & $1.2 \times 10^{-3}$ & - \\
\hline$N_{i t}$ & 2 & $10,50,100,200$ & - & $10,50,100$ & - \\
$\varepsilon_{\text {min }}$ & & $2.1 \times 10^{-5}$ & - & $5.8 \times 10^{-6}$ & - \\
\hline$N_{i t}$ & 1 & $10,50,100$ & - & - & - \\
$\varepsilon_{\text {min }}$ & & $4.4 \times 10^{-4}$ & - & - & - \\
\hline
\end{tabular}

where $e_{i t}$ stands for any of the residuals used to control the iterative error in the flow solvers.

ReFRESCO simulations were performed with three different flow settings: segregated solution with second-order schemes (S2); coupled solution with second order-schemes (C2) and first-order time integration with the segregated solver (S1). All the remaining flow solvers used only one set of settings described above. Table 3 presents the values of $e_{i t}$ used by the solvers that do not limit the number of iterations at each time step together with the number of level tested $N_{s}$. The table also includes the minimum value of $\varepsilon_{\text {min }}$ and the test cases included in the submission. It is recalled that the ANSYS-CFX simulations also included a maximum number of iterations at each time step $N_{i t}=10$.

Tables 4 and 5 present the values of $\varepsilon_{\text {min }}$ for the pimpleFoam and STAR CCM+ simulations after the conversion of $N_{i t}$ to the average value of the maximum residuals, taken as $e_{i t}$. For the pimpleFoam simulations, the decrease of the residuals for the larger time steps (TD and TC) is significantly smaller than for the smaller time steps (TB and TA). On the other hand, the STAR CCM+ results do not show the same trend for all the grids. Therefore, it is not easy to control iterative errors using the number of iterations performed at each time step. In the worst scenario, increasing $N_{i t}$ does not decrease the iterative error if the residual stagnates. Therefore, the values of $\varepsilon_{\min }$ obtained for ANSYS-CFX can be misleading because it is not guaranteed that the residual criterion is satisfied due to $N_{i t}=10$.

The results from all these submissions are assumed to have a sufficient simulation time to obtain a periodic solution with a negligible contribution of the initial condition. However, it was reported that simulations performed with only one or two iterations at each time step may not lead to vortex shedding and so the flow will not become periodic. Naturally, those simulations were not included in the submissions. Plots with all the selected quantities of interest of all the submissions are available 
at [3]. The main trends found in the data are illustrated in the following subsections using $\left(C_{D}\right)_{a v g}$ and $\left(C_{L}\right)_{\max }$.

\subsection{Iterative errors}

The values of $\left(C_{D}\right)_{a v g}$ for G4TD and G3TC are plotted in figure 4 as a function of $\varepsilon$. For these settings, there is at least one simulation from each flow solver. The figure contains two plots for each case: one with $\left(C_{D}\right)_{\text {avg }}$ (left plots) and one with the difference to the solution obtained with the most demanding iterative convergence criterion $\left(\varepsilon_{\min }\right)$ given in percentage of $\left(C_{D}\right)_{\text {avg }}$ calculated with $\varepsilon_{\text {min }}$ (right plots).

The data exhibit similar trends for most flow solvers using implicit time integration except CADYF. As mentioned above, CADYF uses an adaptive time step technique that is influenced by the iterative convergence criteria at each time step and so there is a negligible influence of $e_{i t}$ on the results obtained with this flow solver. On the other hand, the ANSYS-CFX simulations that use several iterative convergence criteria exhibit almost no change of $\left(C_{D}\right)_{\text {avg }}$ for the first three levels of $e_{i t}$, but there is a change of around $3 \%$ for the two lowest levels of $e_{i t}$. However, the existence of a limit of iterations at each time step for these simulations makes the determination of $e_{i t}$ uncertain. For ReFRESCO and STAR CCM+, the values of $\Delta\left(C_{D}\right)_{\text {avg }}$ are larger for the settings that correspond to the largest Courant number (G4TD). The same trend is exhibited by the data obtained in G2TB and G1TA.

The results obtained for the maximum lift coefficient $\left(C_{L}\right)_{\max }$ are depicted in figure 5. Although the trends are similar to those for $\left(C_{D}\right)_{a v g}$, there is a significantly stronger influence of the iterative convergence criteria on $\left(C_{L}\right)_{\max }$ than on $\left(C_{D}\right)_{\text {avg. }}$. A significant amount of results obtained from different flow solvers exhibit $\Delta\left(C_{L}\right)_{\max }$ larger than $10 \%$, as for example the STAR CCM+ results with 10 iterations in each time step and the pimpleFoam solution with 8 or less iterations per time step. Furthermore, the ReFRESCO data with $\varepsilon=10^{-1}$ also show the same level of iterative errors in $\left(C_{L}\right)_{\max }$. In these simulations, the maximum normalized residual of all equations is reduced to values below $5 \times 10^{-4}$, which corresponds to levels below $5 \times 10^{-5}$ for the $\mathrm{L}_{2}$ norm of the residuals. It is worth mentioning that such convergence criterion is more demanding than that used in many unsteady simulations found in the open literature.

The results obtained with DLR-Tau show a weak dependence on $e_{i t}$. However, this compressible flow solver uses an explicit time integration with a dual time step. Therefore, the iterative convergence criterion is less important than in the implicit time integration solvers.

In general, the trends illustrated for $\left(C_{D}\right)_{\text {avg }}$ and $\left(C_{L}\right)_{\text {max }}$ apply to the remaining quantities of interest as well (data available at [3]). The time-averaged base pressure coefficient $\left(C p_{b}\right)_{a v g}$ is a good example, revealing almost the same trends as for $\left(C_{D}\right)_{\text {avg. }}$. The quantities with the smallest influence of the iterative convergence criteria are the Strouhal number $S t$ and the time-averaged angle of flow separation $\left(\theta_{\text {sep }}\right)_{\text {avg }}$. However, as mentioned above, very loose iterative convergence criteria may not lead to vortex shedding in the solution, especially for the largest Courant numbers tested.

\subsection{Influence of iterative errors on the estimation of discretization errors}

The contamination of unsteady flow simulations with iterative errors can also make the estimation of discretization errors troublesome. In the present exercise, it is possible to estimate the exact solution using power series expansions [12] and the data from four grids and time steps that have the same Courant number. Strictly, these data should only be applied when the order of discretization is equal for space and time. However, we will also apply it to the two submissions that used second-order accuracy in space and first-order in time. 

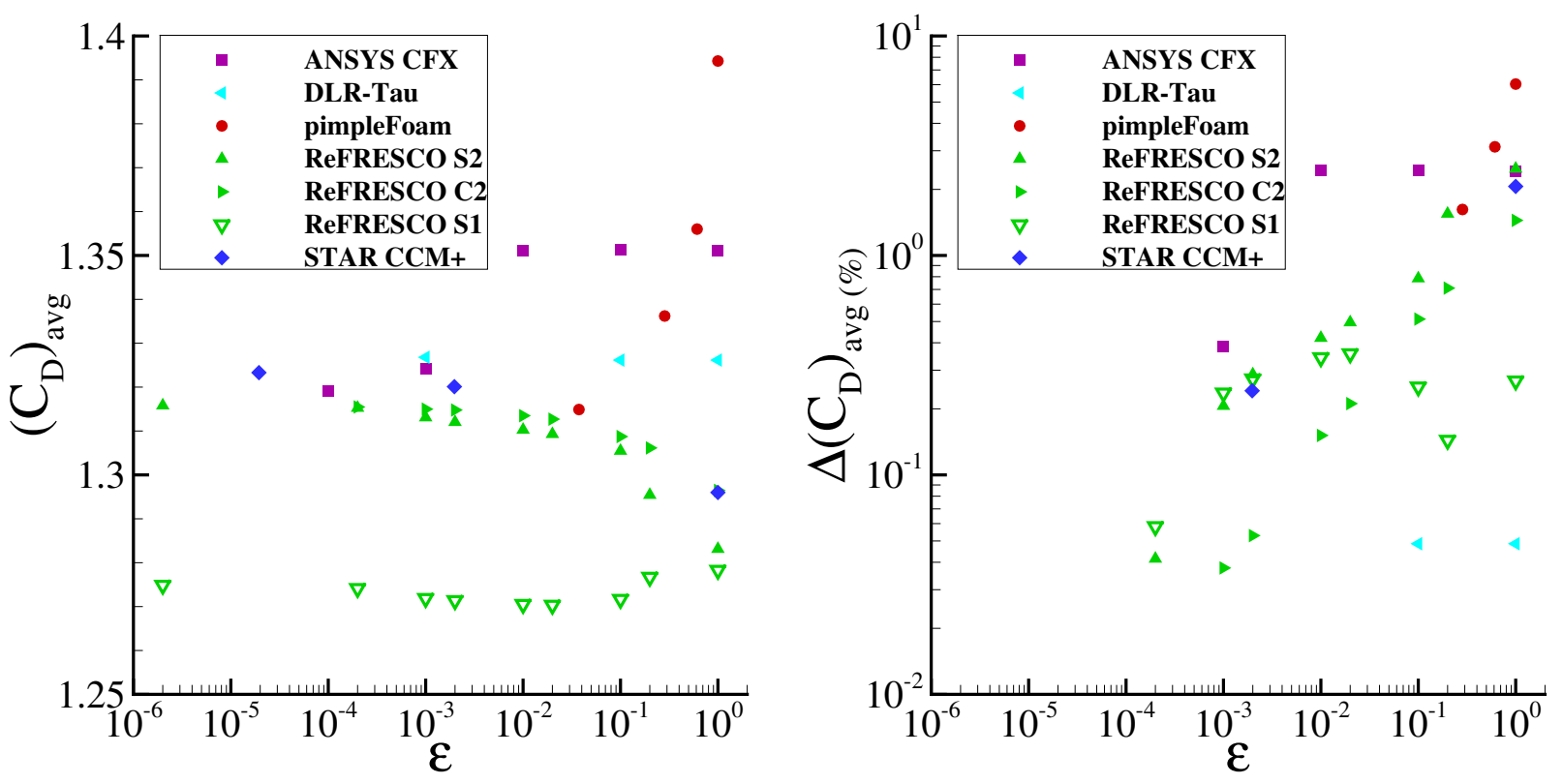

\section{G3TC}
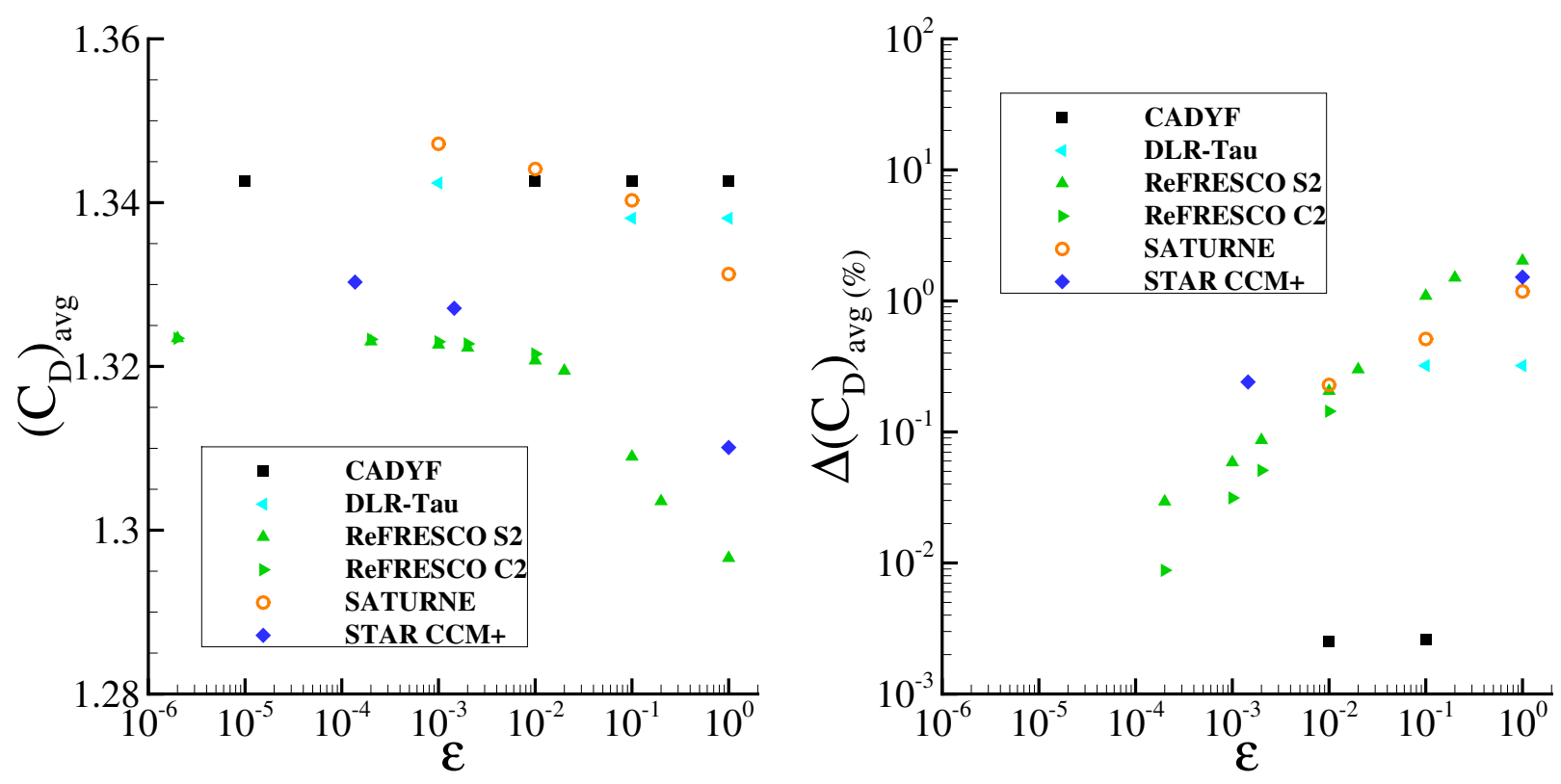

Fig. 4. Time-averaged drag coefficient as a function of the iterative convergence criteria $\varepsilon=e_{i t} /\left(e_{i t}\right)_{\max }$. Right plots present differences to the solution obtained with $\varepsilon_{\text {min }}$ given in percentage of $\left(C_{D}\right)$ avg calculated with $\varepsilon_{\text {min }}$. Coarsest grid G4 with largest time step TD (G4TD) and grid G3 with time step TC (G3TC).

It should be stated that the goal of using the fitting techniques reported in [12] is not to determine the observed order of grid/time convergence or to estimate the exact solution. The aim is to illustrate that discretization error estimation is not independent of iterative errors.

Figures 6 to 9 present the convergence with grid/time refinement of the time-averaged drag coefficient $\left(C_{D}\right)_{\text {avg }}$ for the different submissions. Left plots correspond to the results obtained with the least demanding iterative convergence criteria $\varepsilon_{\max }$ (largest value of $e_{i t}$ ) yet leading to vortex shedding. On the right, the results obtained with the most demanding iterative convergence criteria $\varepsilon_{\text {min }}$ are shown. 
G4TD
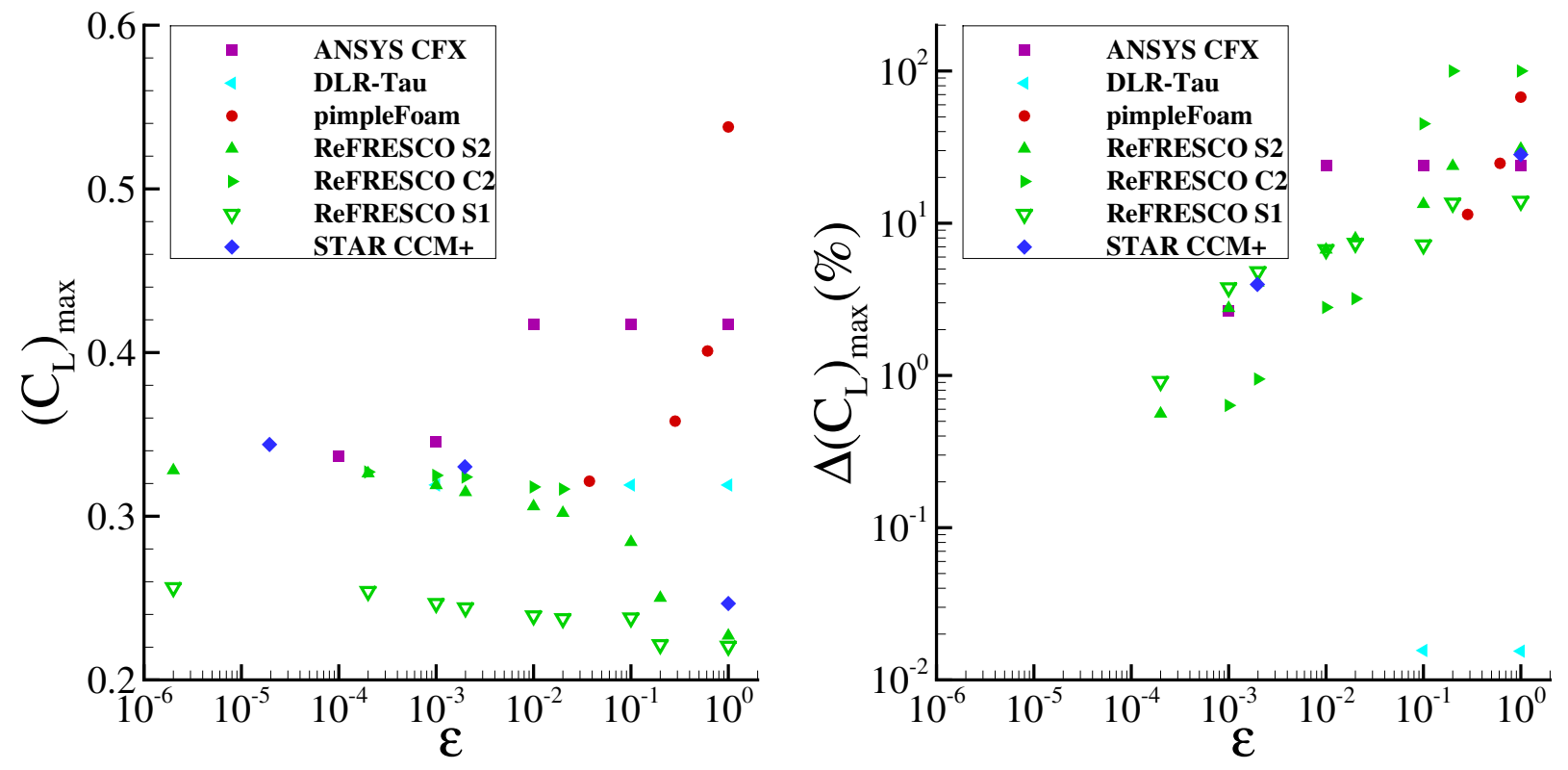

G3TC
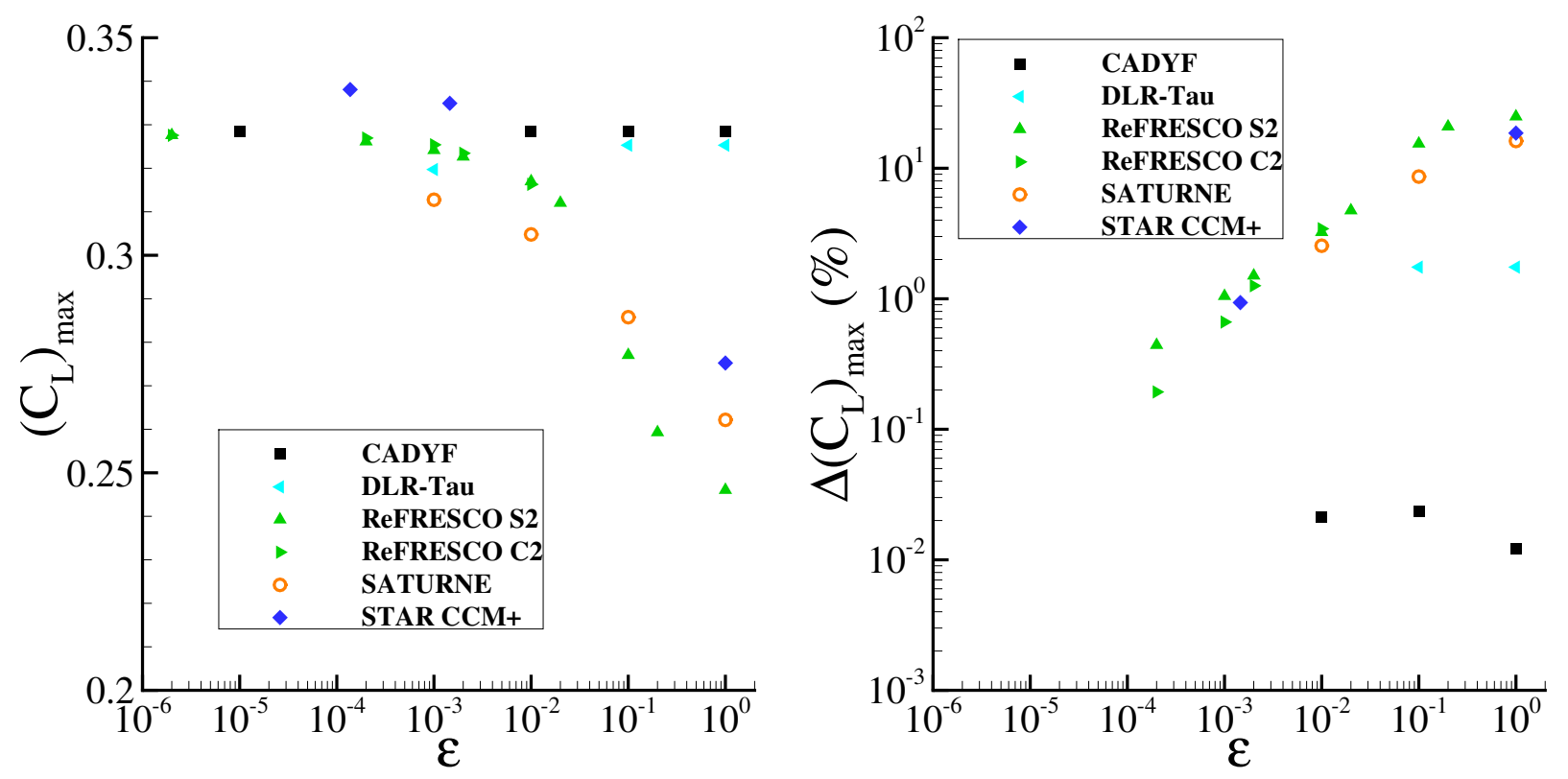

Fig. 5. Maximum lift coefficient as a function of the iterative convergence criteria $\varepsilon=e_{i t} /\left(e_{i t}\right)_{\max }$. Right plots present differences to the solution obtained with $\varepsilon_{\min }$ given in percentage of $\left(C_{L}\right)_{\max }$ calculated with $\varepsilon_{\min }$. Coarsest grid G4 with largest time step TD (G4TD) and grid G3 with time step TC (G3TC).

The influence of the iterative error on the estimation of the discretization error (estimate of the solution for $h_{i} / h_{1}=\Delta t_{i} / \Delta t_{1}=0$.) is evident and tends to increase for increasing Courant numbers. All the incompressible flow solutions are converging monotonically to similar results in the right plots (the exception is the STAR CCM+ data for $\mathrm{Co}_{\max }=15$, but in that case the finest grid solution is most likely still contaminated by iterative errors), whereas the lines fitted to the data obtained with $\varepsilon_{\max }$ exhibit almost an erratic behaviour. The extrapolated solutions to $h_{i} / h_{1}=\Delta t_{i} / \Delta t_{1}=0$ are very similar to the solution obtained with CADYF using grid and time adaptivity. 

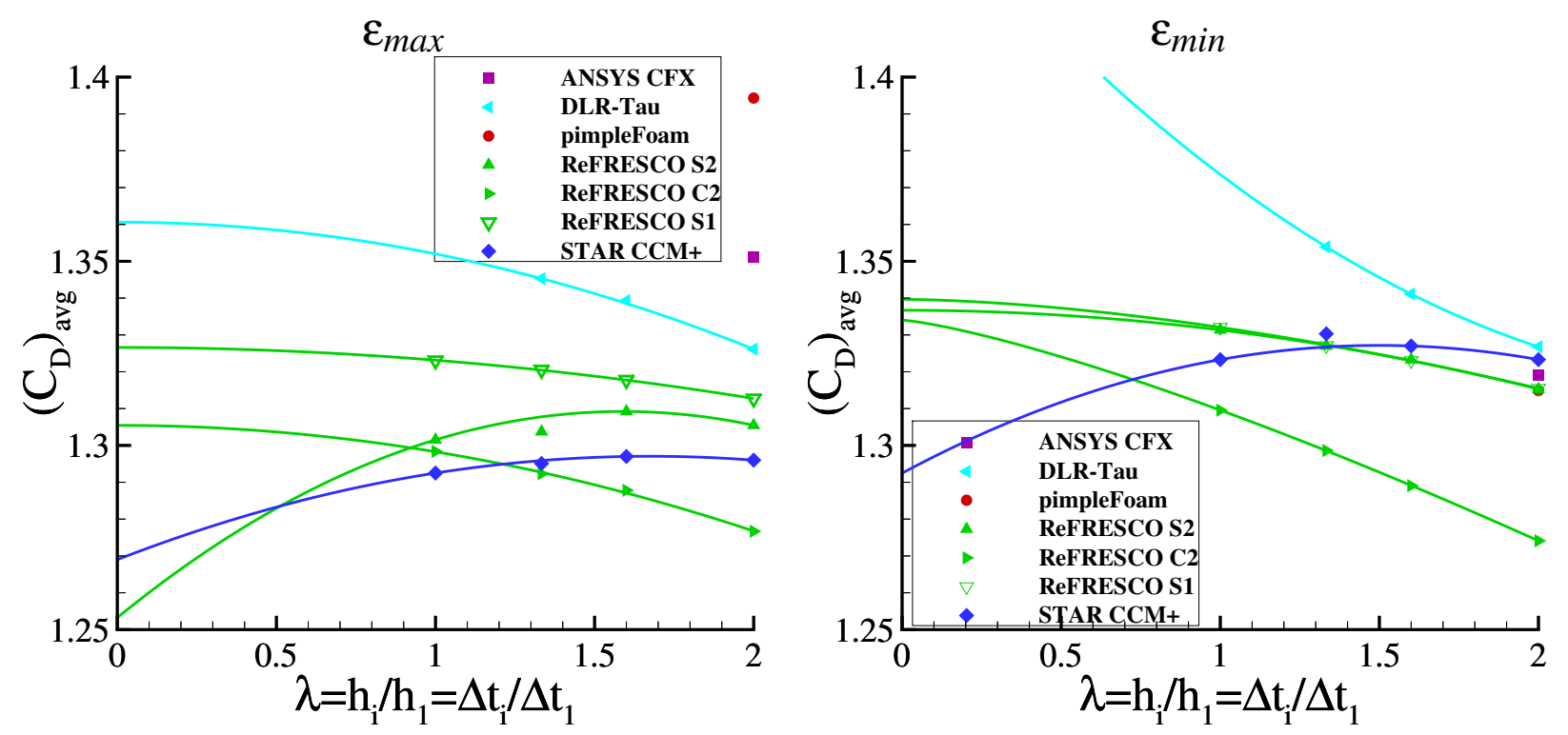

Fig. 6. Time-averaged drag coefficient $\left(C_{D}\right)_{a v g}$ as a function of the grid size $h_{i} / h_{1}$ and time step $\Delta t_{i} / \Delta t_{1}$. Left plots obtained with the least demanding iterative convergence criteria $\varepsilon_{\max }$ and right plots with the most demanding iterative convergence criteria $\varepsilon_{\min } . \mathrm{Co}_{\mathrm{max}}=$ 15.
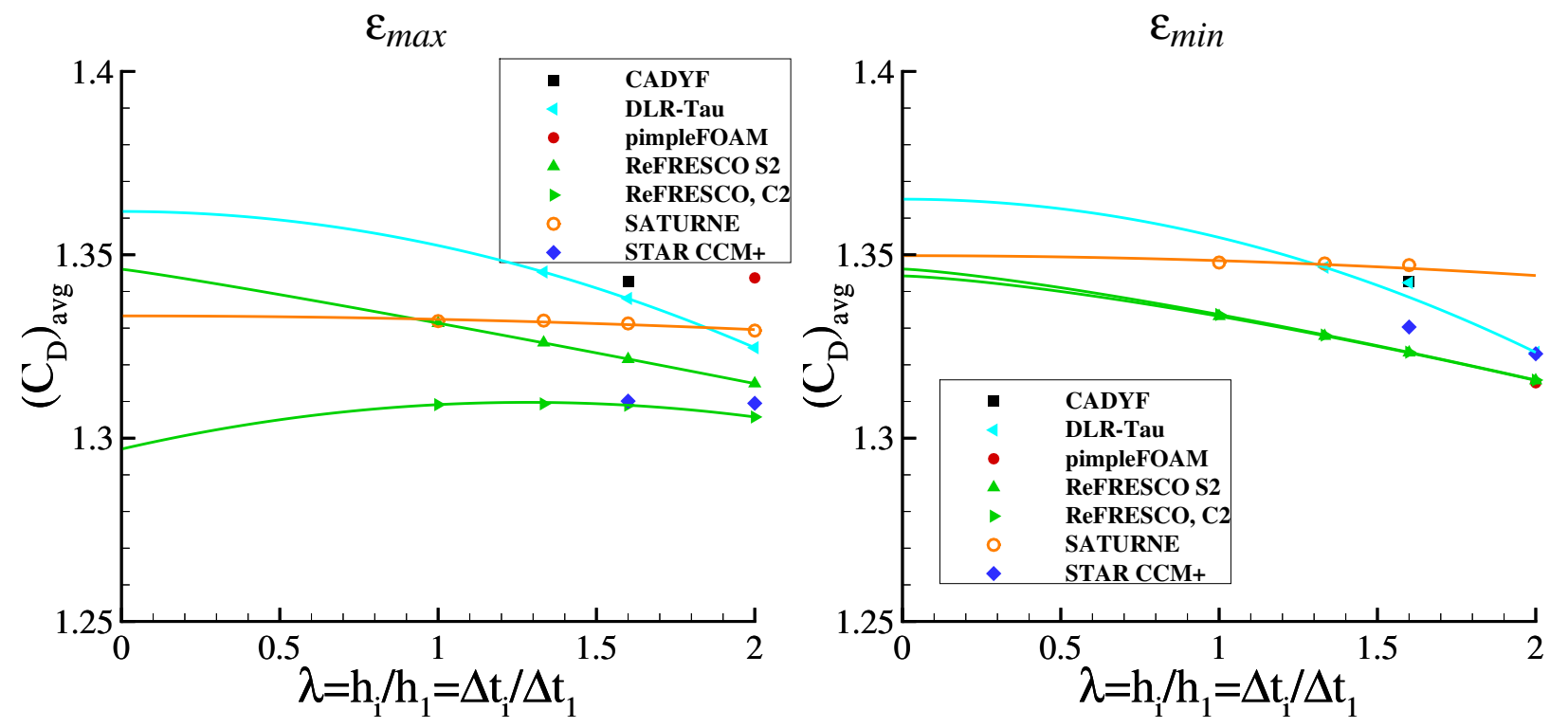

Fig. 7. Time-averaged drag coefficient $\left(C_{D}\right)_{a v g}$ as a function of the grid size $h_{i} / h_{1}$ and time step $\Delta t_{i} / \Delta t_{1}$. Left plots obtained with the least demanding iterative convergence criteria $\varepsilon_{\max }$ and right plots with the most demanding iterative convergence criteria $\varepsilon_{\min } . C o_{\max }=$ 6.

As expected, the DLR-Tau results (explicit time integration) show the smallest influence of $e_{i t}$. Nonetheless, for the highest Courant number $\left(C o_{\max }=15\right.$, figure 6$)$, there is an awkward difference between the line fits for $\varepsilon_{\max }$ and $\varepsilon_{\min }$ respectively that does not appear for the other three cases.

Figures 10 to 13 present the grid/time convergence of the maximum lift coefficient $\left(C_{L}\right)_{\max }$ corresponding to the values of $\varepsilon_{\max }$ and $\varepsilon_{\min }$ of the different submissions. The main trends are similar to those observed for the time-averaged drag coefficient, but with the change of $\left(C_{L}\right)_{\max }$ with $\varepsilon$ significantly larger than that observed for $\left(C_{D}\right)_{\text {avg. }}$. In this case, the extrapolated solutions of all flow solvers (including DLR-Tau) are very similar.

There was one flow solver (ReFRESCO S2) that was used for all the proposed test cases which 

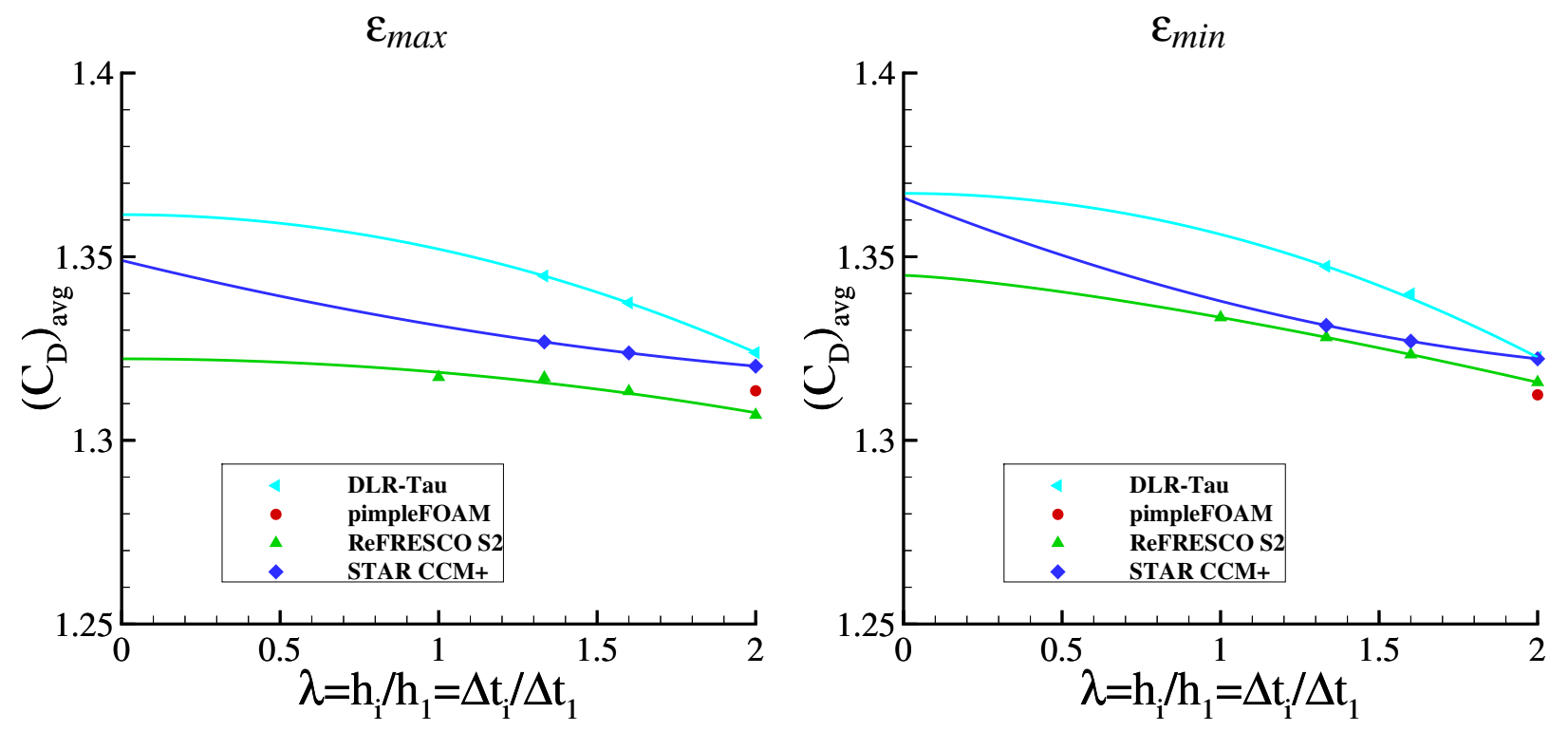

Fig. 8. Time-averaged drag coefficient $\left(C_{D}\right) a v g$ as a function of the grid size $h_{i} / h_{1}$ and time step $\Delta t_{i} / \Delta t_{1}$. Left plots obtained with the least demanding iterative convergence criteria $\varepsilon_{\max }$ and right plots with the most demanding iterative convergence criteria $\varepsilon_{\text {min }}$. $C o_{\text {max }}=$ 1.5 .

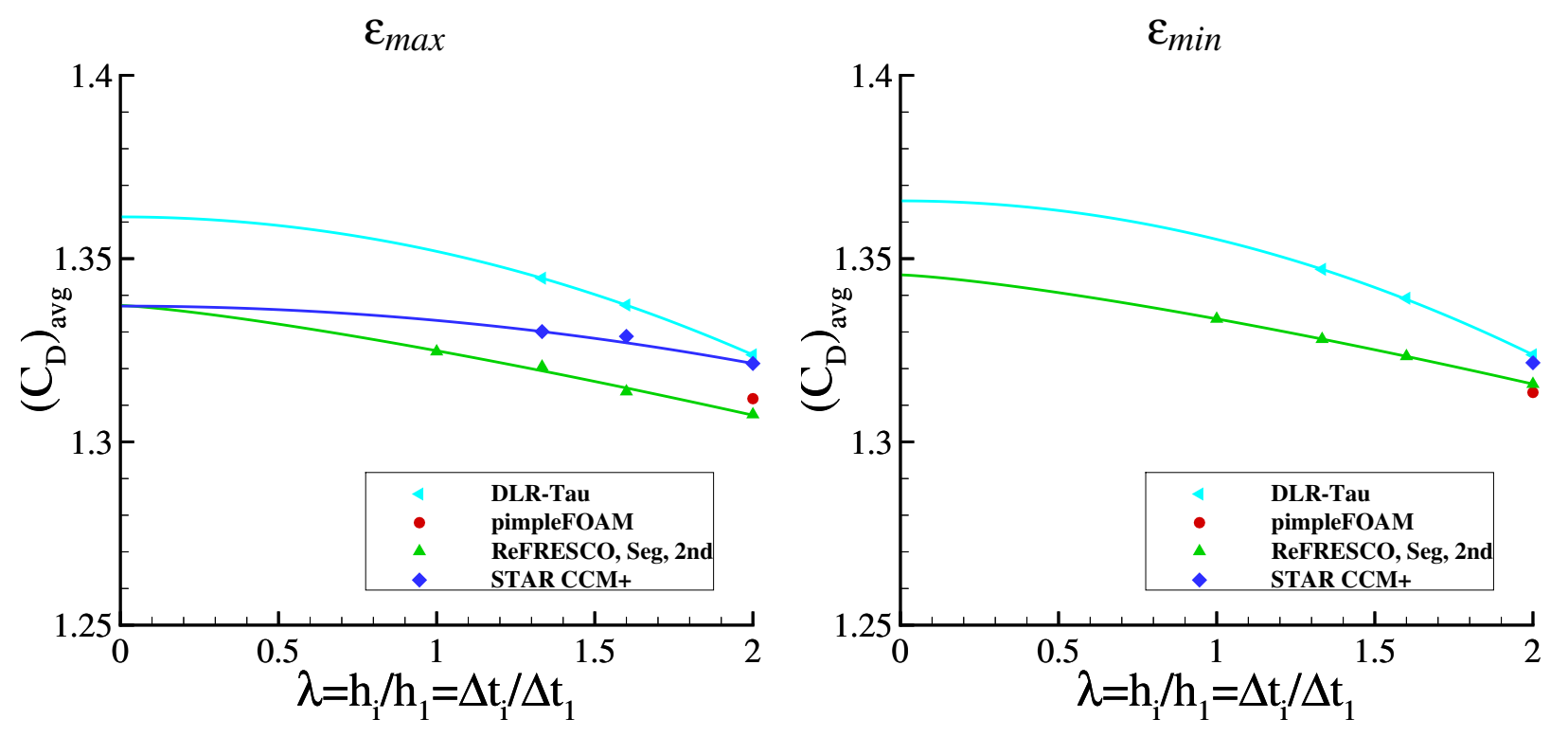

Fig. 9. Time-averaged drag coefficient $\left(C_{D}\right)_{a v g}$ as a function of the grid size $h_{i} / h_{1}$ and time step $\Delta t_{i} / \Delta t_{1}$. Left plots obtained with the least demanding iterative convergence criteria $\varepsilon_{\max }$ and right plots with the most demanding iterative convergence criteria $\varepsilon_{\min }$. $C o_{\max }=$ 0.75 .

makes it possible to illustrate directly the influence of the Courant number on the iterative and discretization errors of $\left(C_{D}\right)_{\text {avg }}$ and $\left(C_{L}\right)_{\max }$. Figures 14 and 15 present the results. The differences between the convergence behaviour obtained with $\varepsilon_{\max }$ and $\varepsilon_{\min }$ increase significantly for the highest Courant numbers. In fact, especially due to the $\left(C_{L}\right)_{\max }$ for the two finest grids (G1 and G2) and the largest Courant number (TD), it is questionable if the iterative convergence criterion is sufficient to obtain a negligible effect on the estimation of the discretization error for these settings. The interaction between $\varepsilon$ and the Courant number is not unexpected, because in an implicit time integration scheme the initial approximation at a given time step is the solution of the previous time step. Therefore, the reduction of the Courant number improves the initial approximation at a given time step. 


\section{Conclusions}

The present paper presents an overview of the 2018 Workshop on Iterative Errors in Unsteady Flow Simulations. The focus of the Workshop was on incompressible flow solvers that typically use implicit time integration, but there was also a submission from a compressible flow solver at a Mach number of 0.2 .

The proposed test case was the two-dimensional flow of an incompressible, Newtonian, single-phase fluid around a circular cylinder at the Reynolds number of 100. Sixteen conditions were proposed including four grid refinement levels and four time steps per grid, thus implying four grid/time refinement studies at constant Courant number. Participants were requested to simulate each case with at least three

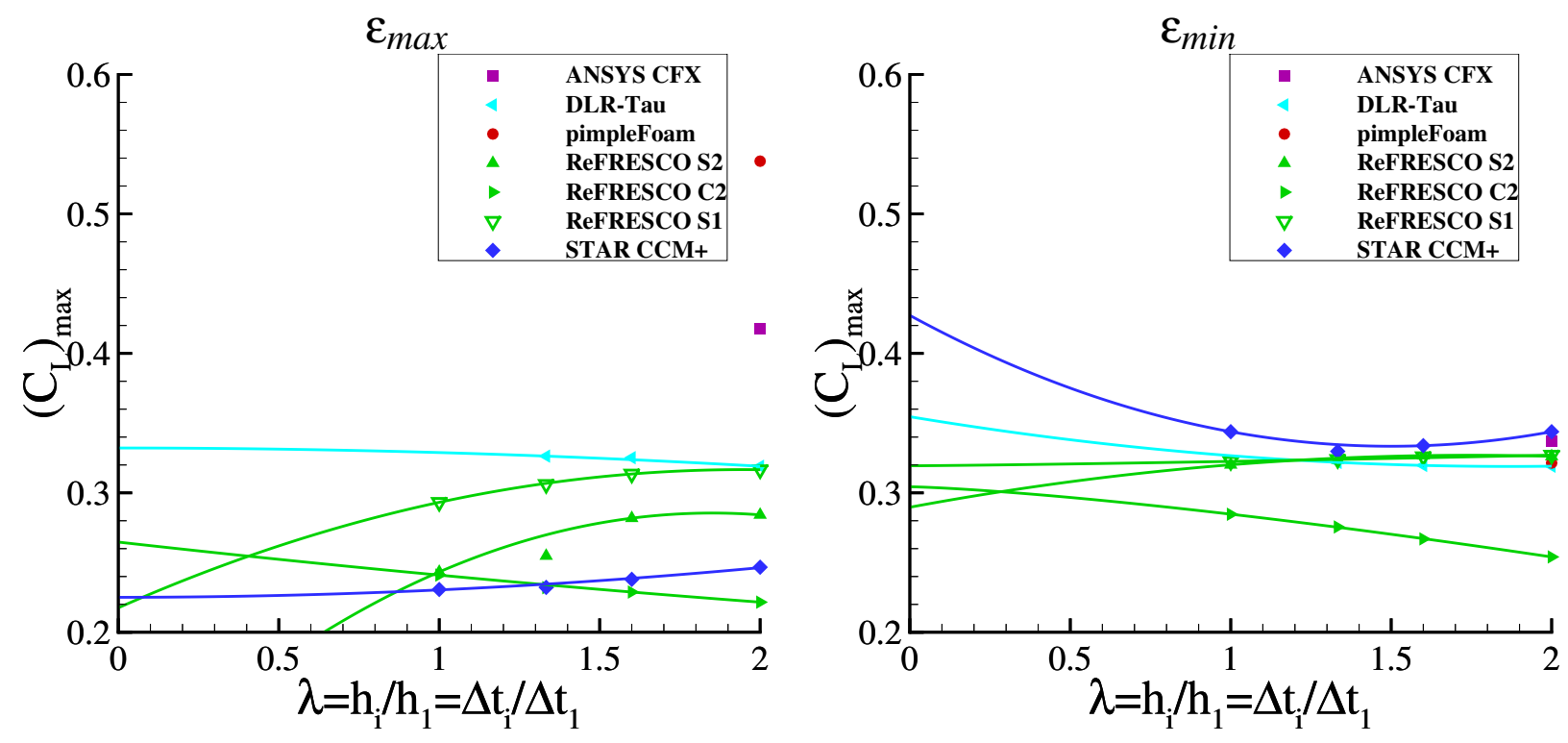

Fig. 10. Maximum lift coefficient $\left(C_{L}\right)_{\max }$ as a function of the grid size $h_{i} / h_{1}$ and time step $\Delta t_{i} / \Delta t_{1}$. Left plots obtained with the least demanding iterative convergence criteria $\varepsilon_{\max }$ and right plots with the most demanding iterative convergence criteria $\varepsilon_{\text {min }}$. Co max $=15$.

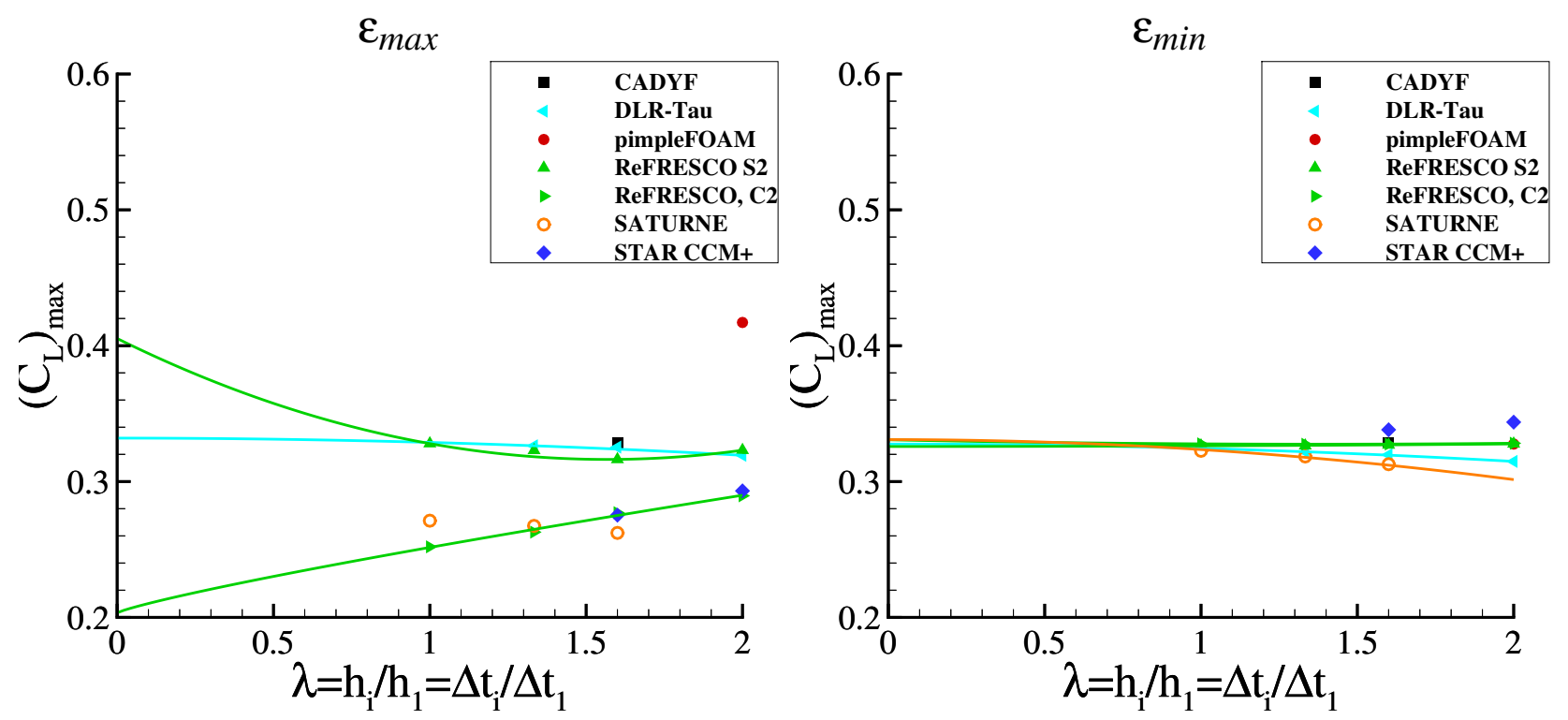

Fig. 11. Maximum lift coefficient $\left(C_{L}\right)_{\text {max }}$ as a function of the grid size $h_{i} / h_{1}$ and time step $\Delta t_{i} / \Delta t_{1}$. Left plots obtained with the least demanding iterative convergence criteria $\varepsilon_{\max }$ and right plots with the most demanding iterative convergence criteria $\varepsilon_{\min }$. $C o_{\max }=6$. 

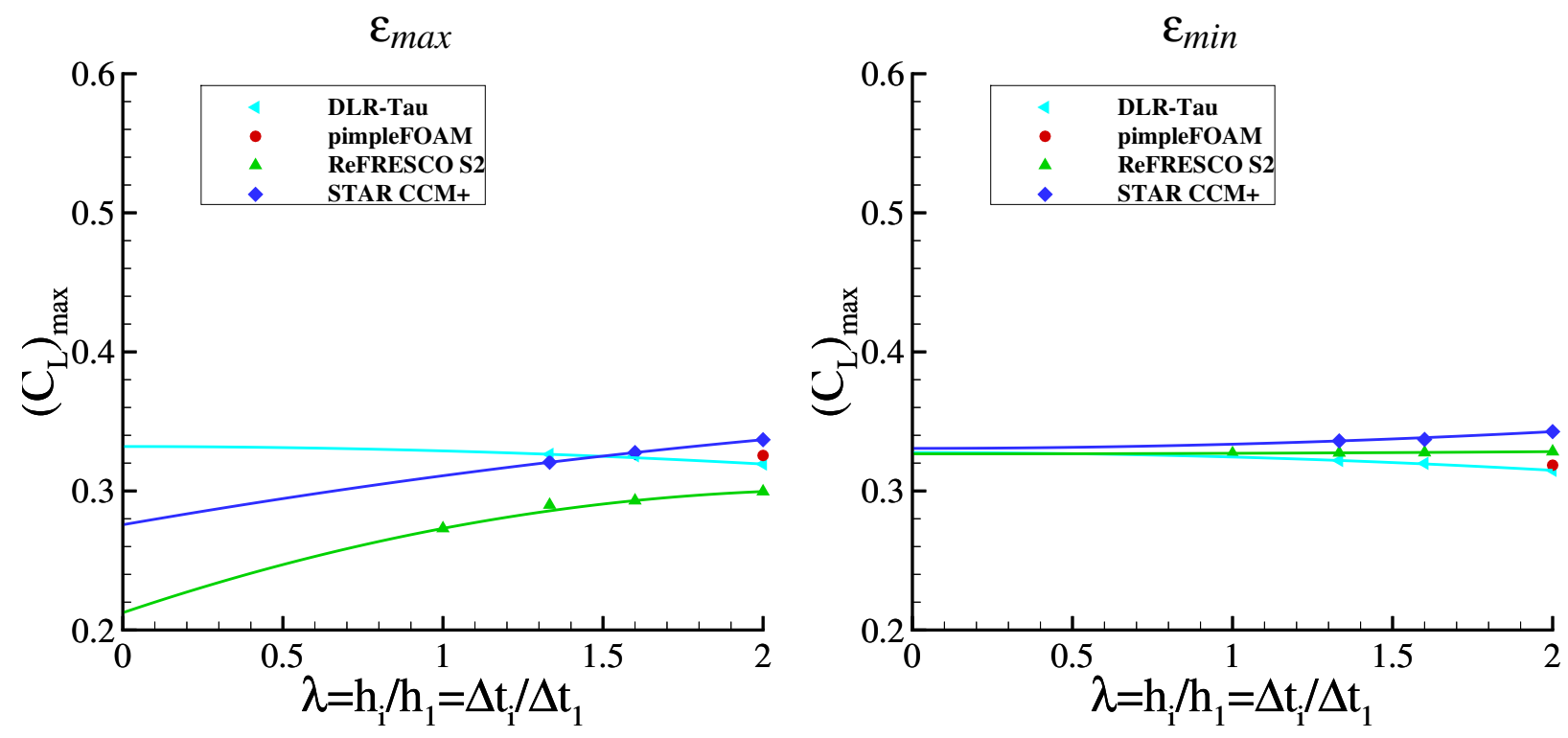

Fig. 12. Maximum lift coefficient $\left(C_{L}\right)_{\max }$ as a function of the grid size $h_{i} / h_{1}$ and time step $\Delta t_{i} / \Delta t_{1}$. Left plots obtained with the least demanding iterative convergence criteria $\varepsilon_{\text {max }}$ and right plots with the most demanding iterative convergence criteria $\varepsilon_{\text {min }} . C o_{\text {max }}=1.5$.
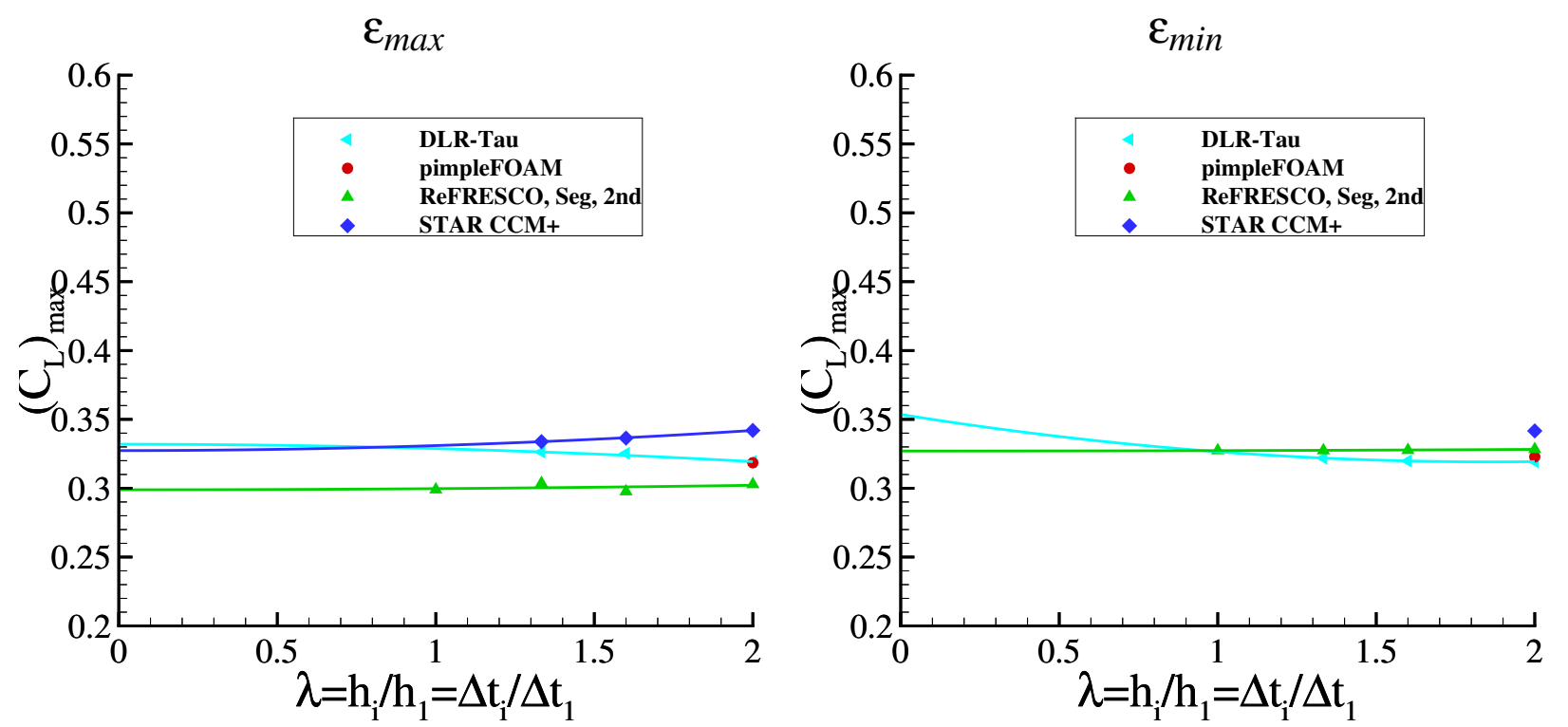

Fig. 13. Maximum lift coefficient $\left(C_{L}\right)_{\max }$ as a function of the grid size $h_{i} / h_{1}$ and time step $\Delta t_{i} / \Delta t_{1}$. Left plots obtained with the least demanding iterative convergence criteria $\varepsilon_{\max }$ and right plots with the most demanding iterative convergence criteria $\varepsilon_{\text {min }}$. $C o_{\text {max }}=$ 0.75 .

different levels of the iterative convergence criteria used at each time step.

Simulations were performed with seven different flow solvers, including five finite volume incompressible solvers using implicit time integration (ANSYS CFX 14.5, pimpleFoam, ReFRESCO, SATURNE, STAR CCM+ v12.06.010-R8), one finite element incompressible flow solver using implicit time integration with grid and time adaptivity (CADYF) and one compressible flow solver using explicit time integration (DLR-Tau).

Quantities of interest included integral/functional and local flow quantities. In this paper, illustrative results are shown for the time-averaged drag coefficient $\left(C_{D}\right)_{\text {avg }}$ and the maximum lift coefficient $\left(C_{L}\right)_{\max }$. The complete set of plots of all data gathered is available at [3]. 

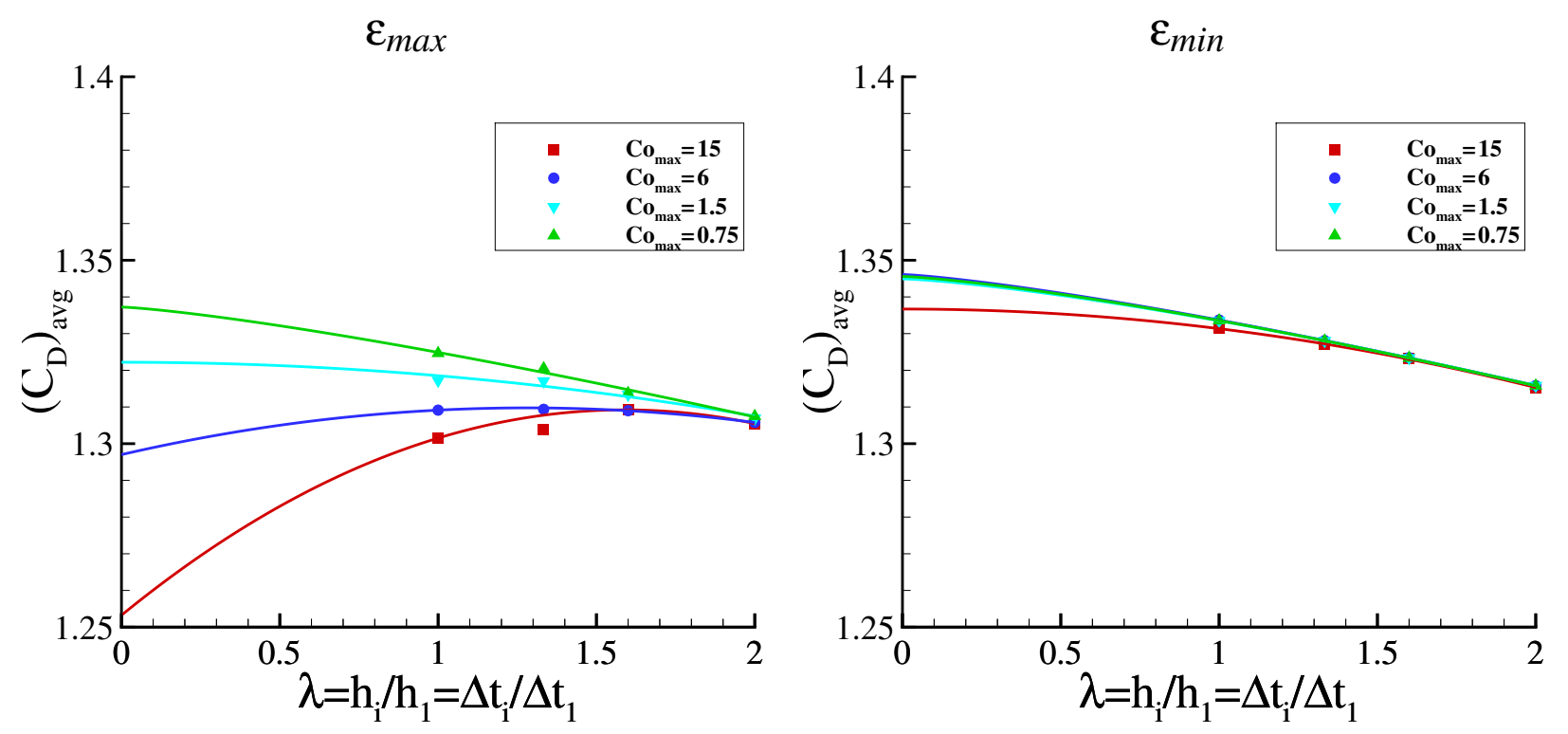

Fig. 14. Time-averaged drag coefficient $\left(C_{D}\right)_{a v g}$ as a function of the grid size $h_{i} / h_{1}$ and time step $\Delta t_{i} / \Delta t_{1}$. Left plots obtained with the least demanding iterative convergence criteria $\varepsilon_{\max }$ and right plots with the most demanding iterative convergence criteria $\varepsilon_{m i n}$. ReFRESCO with second-order schemes in time and space.
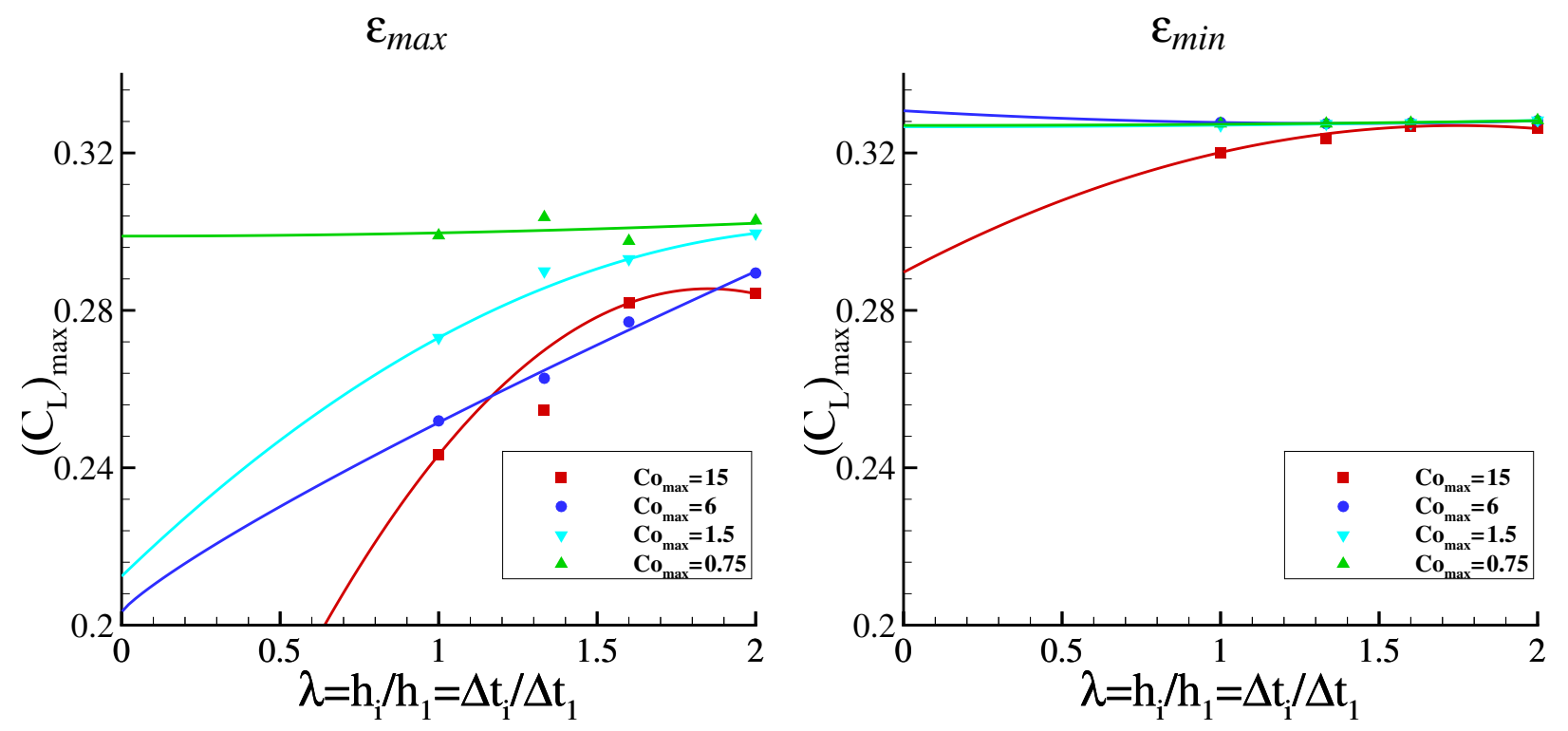

Fig. 15. Maximum lift coefficient $\left(C_{L}\right)_{\max }$ as a function of the grid size $h_{i} / h_{1}$ and time step $\Delta t_{i} / \Delta t_{1}$. Left plots obtained with the least demanding iterative convergence criteria $\varepsilon_{\max }$ and right plots with the most demanding iterative convergence criteria $\varepsilon_{\text {min }}$. ReFRESCO with second-order schemes in time and space.

The main conclusions of this study are summarized below.

- There were two "exceptional" codes among those participating in the Workshop, viz. DLR-Tau and CADYF.

- As expected, DLR-Tau (explicit time integration) exhibited a much weaker dependence on the iterative convergence criteria than the flow solvers using implicit time integration;

- CADYF did not use the proposed grids and time steps due to the grid and time adaptivity techniques included in this flow solver. Its results show a much smaller dependence of the final solution on the iterative convergence criteria than the other incompressible flow solvers. In 
CADYF, iterative errors influence the error estimation performed at each time step; hence the use of a loose iterative convergence criterion reduces the time step (Courant number), decreasing the influence of iterative errors.

- The five flow solvers using implicit time integration with a fixed time step or fixed maximum Courant number exhibited similar trends. However, some of these codes control the iterative error using residuals (ReFRESCO, SATURNE) and others use a fixed number of iterations at each time step (pimpleFoam, STAR CCM+ v12.06.010-R8) or a combination of both (ANSYS CFX 14.5). For the solvers that use a fixed number of iterations, the average value of the maximum residuals at each time step were also reported. Therefore, iterative convergence criteria influence on the solution is illustrated using the ratio between the residuals achieved in a given simulation and the largest residuals used as iterative convergence criteria. The data showed that this simple flow is sufficient to demonstrate the influence of the iterative convergence criteria used at each time step $e_{i t}$ on the numerical error of unsteady flow simulations performed with implicit time integration. Naturally, the influence of $e_{i t}$ on the selected quantities of interest is not always the same. In the present test case:

- The effect of $e_{i t}$ on $\left(C_{L}\right)_{\max }$ is significantly stronger than on $\left(C_{D}\right)_{\text {avg }}$;

- For the same level of $e_{i t}$, the influence of the iterative error diminishes when the Courant number is decreased;

- Although statistical convergence is not illustrated in the present paper, several participants reported that it is possible to statistically converge solutions to levels significantly smaller than the iterative error of the same solutions.

The main goal of these Workshops on Iterative Errors in Unsteady Flow Simulations has been achieved. Iterative errors must be carefully addressed in unsteady flow simulations performed with implicit time integration. The use of loose iterative convergence criteria, often justified by the cost of the simulation time, may lead to misleading results, even though apparently acceptable statistical and/or discretization convergence levels were achieved. In such cases, the numerical error may be dominated by the iterative error, which may exhibit levels dependent on the selected quantity of interest.

\section{Acknowledgements}

The participants of the 2017 Workshop, in particular A. Jones, S. Guda and I. Çelik are gratefully acknowledged for their essential contribution to the organization of the second edition reported in this paper. The Laboratory for Advanced Computing at University of Coimbra (http://www . lca . uc. pt) is acknowledged for providing computing resources to perform some of the submissions of this Workshop.

\section{References}

[1] Eça L., Vaz G, Toxopeus S., Hoekstra M. - Numerical Errors in Unsteady Flow Simulations - J. Verif. Valid. Uncert. Jun 2019, 4(2): 021001.

[2] http://web.tecnico.ulisboa.pt/ist12278/Workshop_iterative_ 2017.htm

[3] http://web.tecnico.ulisboa.pt/ist12278/Workshop_iterative_ 2018/Workshop_iterative_2018.htm

[4] ANSYS CFX http://www.ansys.com/Products/Fluids/ANSYS-CFX

[5] A.Hay, S.Etienne, D.Pelletier, A.GaronA. Hay, D. Pelletier - hp-Adaptive time integration based on the BDF for viscous flows - Journal of Computational Physics, Volume 291, 15 June 2015, Pages 151-176. 
[6] D. Schwamborn, T. Gerhold, R. Heinrich - The DLR TAU-CODE: Recent Applications in Research and Industry - European Conference on Computational Fluid Dynamics, ECCOMAS CFD 2006, Netherlands 2006.

[7] OpenFoam http://www.openfoam.com/

[8] ReFRESCOhttp://www.refresco.org/

[9] SATURNE/http://code-saturne.org/cms/

[10] STAR-CCM+ https://mdx.plm.automation.siemens.com/

star-ccm-plus

[11] https://www.pardiso-project.org/

[12] Eça L., Hoekstra M. - A Procedure for the Estimation of the Numerical Uncertainty of CFD Calculations Based on Grid Refinement Studies, Journal of Computational Physics, Volume 262, 2014, pp. 104-130. 